\title{
Two-Year Effects of Interdisciplinary Intervention for Hip Fracture in Older Taiwanese
}

\author{
Yea-Ing L. Shyu, PhD, * Jersey Liang, PhD, ${ }^{\dagger \neq}$ Chi-Chuan Wu, MD, ${ }^{\mathcal{S}}$ Juin-Yih Su, MD," \\ Huey-Shinn Cheng, MD, ${ }^{\#}$ Shib-Wei Chou, MD, PhD, ${ }^{* *}$ Min-Chi Chen, PhD, ${ }^{\dagger \dagger}$ Ching-Tzu Yang, MSN ${ }^{\ddagger}$ \\ and Ming-Yueh Tseng, MSN
}

OBJECTIVES: To explore the 2-year outcomes of an interdisciplinary intervention for elderly patients with hip fracture. DESIGN: Randomized experimental design.

SETTING: A 3,000-bed medical center in northern Taiwan. PARTICIPANTS: Patients with hip fracture $(\mathrm{N}=162)$ : 80 in the intervention group and 82 in the usual care control group. INTERVENTION: An interdisciplinary program of geriatric consultation, continuous rehabilitation, and discharge planning.

MEASUREMENTS: Outcomes (clinical outcomes, self-care ability, health-related quality of life (HRQoL), service utilization, and depressive symptoms) were assessed 1, 3, 6, 12, 18 , and 24 months after discharge. Self-care ability (ability to perform activities of daily living (ADLs)) was measured using the Chinese Barthel Index. HRQoL was measured using the Medical Outcomes Study 36-item Short Form Survey, Taiwan version (SF-36). Depressive symptoms were measured using the Chinese Geriatric Depression Scale, short form.

RESULTS: Subjects in the intervention group had significantly better ratios of hip flexion $(\beta=5.43, P<.001)$, better performance on ADLs $(\beta=9.22, P<.001)$, better recovery of walking ability (odds ratio $(\mathrm{OR})=2.23, P<.001$ ), fewer falls $(\mathrm{OR}=0.56, P=.03)$, fewer depressive symptoms $(\beta=$ $-1.31, P=.005)$, and better SF-36 physical summary scores $(\beta=6.08, P<.001)$ than the control group during the first 24 months after discharge. The intervention did not affect the peak force of the fractured limb's quadriceps, mortality, service utilization, or SF-36 mental summary score.

From the *School of Nursing, ${ }^{\dagger \dagger}$ Department of Public Health and Biostatistics Consulting Center, and ${ }^{\ddagger \ddagger}$ Graduate Institute of Nursing, Chang Gung University, Taoyuan, Taiwan; 'School of Public Health and ${ }^{\ddagger}$ Institute of Gerontology, University of Michigan, Ann Arbor, Michigan; ${ }^{\S}$ Trauma Division, Department of Orthopedics and Departments of "Internal Medicine and

**Physical Medicine and Rehabilitation, Chang Gung Memorial Hospital, Taoyuan, Taiwan; and "Department of Orthopedics, Chang Gung Memorial Hospital, Keelung, Taiwan.

Address correspondence to Yea-Ing L. Shyu, School of Nursing, Chang Gung University, 259 Wen-Hwa 1st Road, Kwei-Shan, Taoyuan 333, Taiwan.

E-mail: yeaing@mail.cgu.edu.tw

DOI: 10.1111/j.1532-5415.2010.02882.x
CONCLUSION: The interdisciplinary intervention for hip fracture benefited elderly persons with hip fracture by improving clinical outcomes, self-care ability, and physical health-related outcomes and by decreasing depressive symptoms during the first 24 months after hospital discharge. J Am Geriatr Soc 58:1081-1089, 2010.

Key words: hip fracture; older adults; interdisciplinary intervention; self-care ability; walking ability; healthrelated quality of life

$\mathrm{H}$ ip fracture has been found to severely affect the functional and health outcomes of elderly persons, ${ }^{1-6}$ but few studies have examined the trajectory of functional recovery over the year after hip fracture. In one study of patients followed for 2 years after hip fracture, the degree and timing of recovery were found to vary according to functional domain, but many of these domains remained below prefracture levels, even at 2 years. ${ }^{7}$ In that study, patients who had been independent before the fracture were found to develop new dependencies 12 and 24 months after hip fracture. Thus, the effect of hip fracture on physical outcomes can be observed for at least 2 years after the fracture.

Elderly patients with hip fracture have been shown to benefit from postoperative rehabilitation, early discharge planning programs, and transitional care programs, ${ }^{8,9}$ but the majority of these studies have analyzed data from developed Western countries, and few have examined the longitudinal effects of interventions more than twice within the first year after discharge. Less is known about the effects of interventions up to 2 years for elderly patients with hip fracture in Asian countries. At the same time, few studies have examined intervention effects on performance-based and self-reported outcome indicators. In addition, Taiwan differs substantially from developed Western countries in its healthcare system, clinical practice, case mix, culture, and social organization. These differences make it necessary to 
validate Western-based studies on the effects of intervention programs for older adults with hip fracture in Taiwan.

Older Taiwanese with hip fracture were previously examined in a randomized intervention study on the effects of an interdisciplinary intervention program for these older adults during the first postdischarge year. ${ }^{10,11}$ That program consisted of geriatric consultation, continuous rehabilitation, and discharge planning. The intervention program was found to benefit older patients with hip-fracture by improving their clinical outcomes and self-care abilities and by decreasing depressive symptoms. The purpose of this article is to report the long-term effects of this intervention program on self-reported and performancebased outcome variables from the first to second year after patient discharge. It was hypothesized that the benefits of the interdisciplinary intervention program would continue from the first to second year after discharge.

\section{METHODS}

\section{Participants}

Inclusion criteria for subjects were aged 60 and older, admitted to hospital for an accidental single-side hip fracture, receiving hip arthroplasty or internal fixation, able to perform full range of motion against gravity and against some or full resistance, a prefracture Chinese Barthel Index (CBI) score greater than 70, and living in northern Taiwan. Patients were excluded if they were severely cognitively impaired, making them unable to follow orders (score $<10$ on the Chinese Mini-Mental State Examination), ${ }^{12}$ or terminally ill.

Participants $(\mathrm{N}=162: 80$ in the intervention group, 82 in the control group) were recruited from September 2001 to November 2004 and followed for 2 years (Figure 1). At the end of the second year, 55 subjects remained in the intervention group and 48 in the control group. Nine subjects in the intervention group and 13 in the control group died during the 2-year follow-up. More subjects died during the second year (5 in the intervention group and 7 in the control group) than during the first year. In the intervention group, 16 subjects refused to continue participating and dropped out during the first year; no more dropped out in the second year. In the control group, 21 subjects dropped out: 14 in the first year and seven in the second year. For a previous study, ${ }^{10}$ a sample size of 65 subjects in each group was required to provide a power of 0.80 and alpha of 0.05 for a medium effect size of $0.50^{13}$ in terms of ADL improvement from postsurgical discharge to 3 months later. It was assumed that the intervention program might be most effective during the first 3 months because of greater recovery in this period. ${ }^{6}$ To allow for potential dropouts, it was therefore decided to recruit approximately 80 subjects in each group.

\section{Intervention Program}

The interdisciplinary intervention program included geriatric consultation services, a continuous rehabilitation program, and discharge-planning services ${ }^{10,11}$ (Appendix 1). The geriatric consultation was designed to detect potential medical and functional problems and to decrease delays before surgery and was administered by a geriatrician and geriatric nurses during hospitalization before and after surgery. Geriatric consultations were based on geriatric assessments and provided to the surgeon in charge to suggest time of surgery, use of infection and thromboembolic prophylaxis, postoperative nutrition management, urinary tract management, and delirium prevention and management.

Continuous rehabilitation was designed to provide early postoperative rehabilitation, facilitate mobility, plan for hospital discharge, and provide rehabilitation in the patient's usual environment; geriatric nurses and physical therapists delivered this component. In this component of the intervention, the inpatient rehabilitation program included a hip fracture-oriented intervention and a general intervention program for enhancing physical fitness. The at-home rehabilitation program included a hip fracture-oriented intervention and a general intervention program for enhancing physical fitness. The exercise protocol emphasized ankle dorsiflexion with knee extension, isometric full-knee extension, gently bouncing vertical jump with knee semiflexed and foot on the floor, and ball-rolling activities to enhance proprioception, depending on each patient's condition.

The discharge planning component was designed to maintain continuity of care and to assure appropriateness of referrals and was delivered by geriatric nurses. Predischarge assessment included caregiver's competence, resources, family function, patient's self-care ability, and needs for community or long-term care services, with necessary referrals made. The home environment was assessed, environmental modifications were suggested, and followup adherence was monitored.

In summary, each participant in the intervention group received one geriatrician visit, a mean of $5.4 \pm 2.4$ geriatric nurse visits, a mean of $3.1 \pm 1.6$ physical therapist visits, and one rehabilitation physician visit during the hospital stay. After discharge, each participant in the intervention group received an average $9.9 \pm 2.3$ geriatric nurse visits and an average $3.0 \pm 1.1$ physical therapist visits at home.

\section{Usual Care}

After an injurious fall, patients are usually sent directly to the hospital emergency department, although some patients may visit an outpatient clinic, through which they enter the hospital. Orthopedists care for these patients. Consultations for internal medicine care are occasionally made depending on the patient's condition. Consultations with anesthesiologists are sometimes made. Before surgery, routine examinations include X-ray, electrocardiogram, testing for blood chemistry, and blood cell counts. Skin traction before surgery is usually performed. Patients then receive internal fixation or arthroplasty. After surgery, patients usually stay in the recovery room for vital signs to be monitored. After vital signs have become stable, patients are transferred to the trauma or orthopedic wards. In the first 2 to 3 days, nurses teach patients how to exercise while still in bed, and caution is taken while changing their position. Pain relief medications are also administered. Antibiotics are administered for 2 or 3 days. Hemobags are removed on the third day, and physical therapy usually starts the same day. Participants in the control group received an average of $1.1 \pm 1.6$ sessions of physical therapy.

Patients are usually discharged from the hospital approximately 5 to 7 days after surgery. After hospital discharge, no provision is made for at-home rehabilitation or nursing care. 


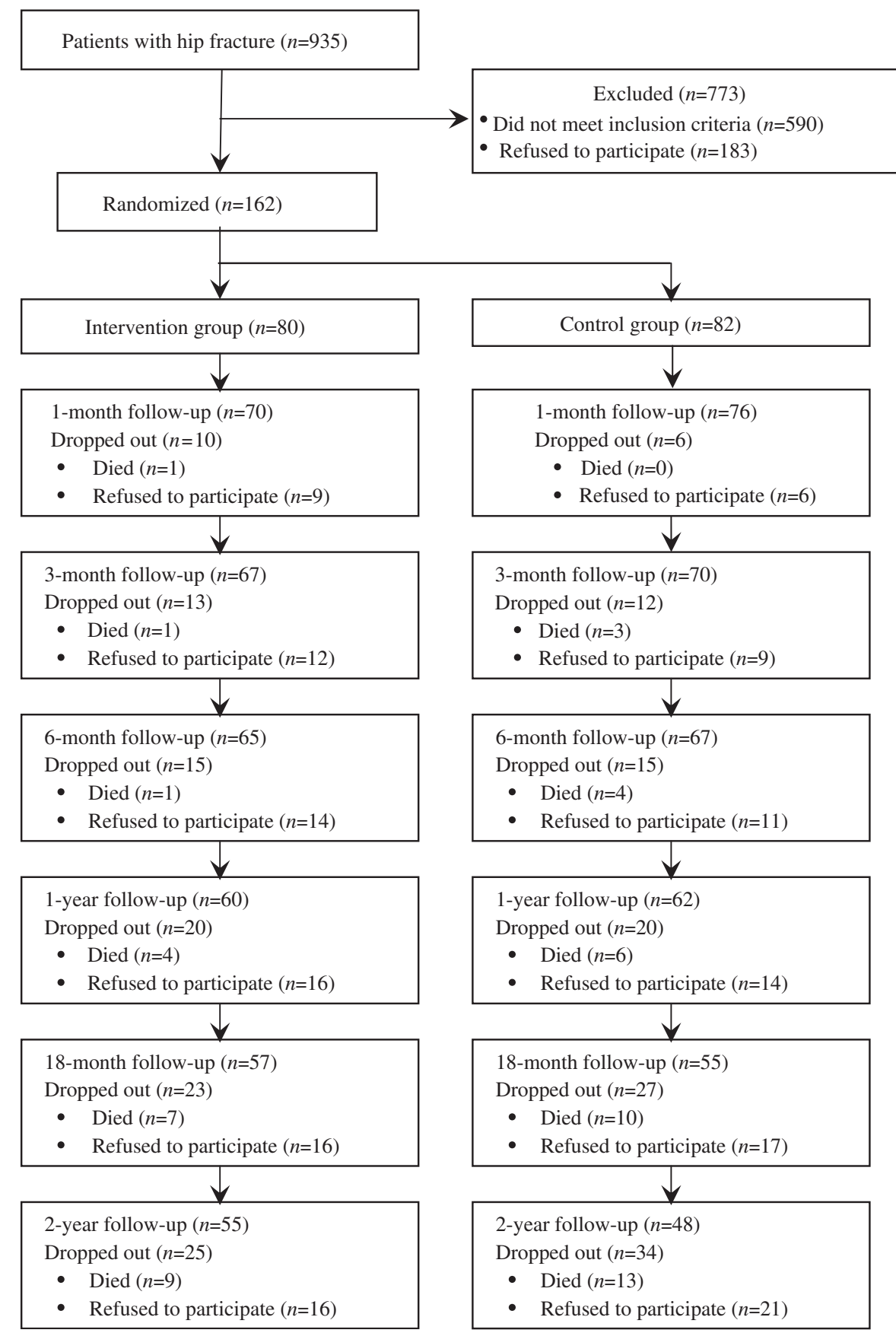

Figure 1. Flow diagram of study.

No participants in the control group received at-home physical therapy. In general, adherence to the medical follow-up schedule is poor. Telephone follow-up is seldom provided, and home environments are not assessed. Patients and their families are generally ignorant of rehabilitation, and little health education is offered.

\section{Outcome Variables}

Outcomes were measured using performance-based and self-report measures. Performance-based measures included ratio of hip flexion (RHF), peak force of the fractured limb's quadriceps, recovery of walking ability, self-care ability, and occurrence of falls. Self-report measures included health-related quality of life (HRQoL) and depressive symptoms. In addition, the intervention and control groups were compared for mortality and service utilization, including hospital readmission, emergency department visits, and institutionalization.

\section{Performance-Based Measures}

RHF was defined as the range of motion (ROM) of the affected hip joint divided by the ROM of the unaffected hip joint. The peak force of the fractured limb's quadriceps was measured using MICROFET2, a portable force-evaluation and testing device (Hoggan Health Industries, Inc., Draper, UT).

Recovery of walking ability was rated during face-toface interviews using the walking ability item in the CBI ${ }^{14}$ and comparing it with prefracture walking ability (rated at 
admission using the same item). Raters asked subjects to walk for 50 yards. Walking ability was rated from 0 to 15 , with the following categories: 0 (immobile or $<50$ yards), 5 (wheelchair independent, including corners, $>50$ yards), 10 (walks with verbal or physical help of one person, $>50$ yards), and 15 (independent, but may use any aid, e.g., a cane, $>50$ yards). Recovery of walking ability to the prefracture level was rated 1 ; no or less recovery than the prefracture level was rated 0 .

\section{Self-Report Measures}

HRQoL was measured using the physical (PCS) and mental (MCS) component summary scales of the Taiwan version of the Medical Outcomes Study 36-item Short Form Survey (SF-36). ${ }^{15-17}$ The SF-36 consists of 36 items representing health concepts, including physical functioning, role disability due to physical health problems, bodily pain, vitality, general health perceptions, social functioning, role disability due to emotional problems, and general mental health. PCS and MCS scores were calculated using norm-based (50, 10) scoring methods ${ }^{18}$ with Taiwan-specific SF-36 algorithms. ${ }^{16}$ Scores in each scale range from 0 to 100 , with higher scores representing better health outcomes.

Depressive symptoms were assessed using the Chinese version of the Geriatric Depression Scale, short form (GDSs). ${ }^{19,20}$ The maximum GDS-s score is 15 ; higher scores indicate more-severe depressive symptoms. Patients with a score of 5 or higher were categorized as being at risk for a clinical diagnosis of depression. The sensitivity of the GDS-s score to the intervention was maximized by using it as a continuous variable in the longitudinal outcome analysis to indicate the severity of depressive symptoms. Reliability (internal consistency) and construct validity of the GDS-s have been established in older Taiwanese. ${ }^{20}$ The reliability for this study ranged from 0.81 to 0.87 .

\section{Self-Care Ability Measure}

Self-care ability was measured using the $\mathrm{CBI}^{14}$ in terms of activities of daily living (ADLs: eating, transferring, grooming, toileting, bathing, walking, climbing stairs, dressing, bowel and bladder control). With scores ranging from 0 to 100 , the CBI has been shown to have satisfactory reliability and validity for assessing frail older adults in Taiwan. ${ }^{14}$

CBI scores were obtained using observation of performance and patient self-report, except for prefracture status which was based on patients' recall of their prefracture performance of ADLs at admission. At 1, 3, 6, 12, 18, and 24 months after discharge, 50-yard walking and transferring abilities were assessed by observing patients perform these activities. All other activities (eating, grooming, toileting, bathing, climbing stairs, dressing, and bowel and bladder control) were rated according to patient self-report.

\section{Procedures}

Human subject approval was obtained before collecting data. An institutional ethics panel reviewed the protocol, and signed consent was obtained from patients before collecting data. Research assistants who screened the admission list twice a day recruited subjects from the emergency department. Those who agreed to participate were randomly assigned to an intervention or control group. Subjects in the intervention group then received routine hospital care plus the intervention program, whereas subjects in the control group received only routine hospital care plus regular social contact provided by a research nurse at the same time that the intervention group received the intervention. All subjects were assessed at 1, 3, 6, 12, 18, and 24 months after discharge for clinical outcomes, service utilization outcomes, self-care ability, and depressive symptoms. The subjects were blinded, but the evaluators were not.

\section{Statistical Analysis}

Analyses were performed using an intention-to-treat principle. Differences in baseline characteristics, including prefracture self-care ability of the intervention and control groups, were assessed using two-sample $t$-tests or chi-square tests, with the significance level set to .05 . To evaluate the effects of the interdisciplinary intervention and to account for correlations in repeated observations over time, a generalized estimating equation (GEE) approach was used. ${ }^{21,22}$ The GEE can account for possible correlations in repeated measures over time and can explore differences at different time points. One advantage of using the GEE model is that partial information can also be used (e.g., data from those who drop out can still contribute to the estimation parameters). This approach is especially useful in longitudinal studies in which sample attrition is inevitable, but the data belonging to study participants who die or drop out within 2 years of discharge can be included in the analysis. For a given outcome variable, the GEE model includes treatment $(1=$ intervention group, $0=$ control group) as a predictor and five dummy variables representing the timing of measurements at 3, 6, 12, 18, and 24 months after discharge $(1=$ measurements at $3,6,12,18$ and 24 months, $0=$ measurement at first month). GEE analyses were performed using SAS Win 8.0 (SAS Institute, Inc., Cary, NC).

Subjects who dropped out $(\mathrm{n}=59)$ were comparable with those remained in the study in terms of age, sex, educational background, type of surgery, length of hospital stay, and number of comorbid conditions. Comparison of subjects who dropped out in the intervention group $(\mathrm{n}=25)$ and in the control group $(n=34)$ showed no significant differences in age, sex, educational background, type of surgery, length of hospital stay, or prefracture self-care ability, so the influence of death and attrition was probably limited. All data were analyzed using SAS software.

\section{RESULTS}

\section{Baseline Participant Characteristics}

Sample characteristics for the intervention and control groups are shown in Table 1 . Of the 162 participants in the final sample, $68.5 \%$ were female, with an average age of $78.2 \pm 7.8$. Fifty-two percent were married, $48.8 \%$ were illiterate, $63 \%$ received internal fixation, and $37 \%$ received arthroplasty. Participants' mean CBI score before fracture was $96.1 \pm 6.5$, representing independence in performing ADLs, and $84.6 \%$ could walk independently. The intervention and control groups did not differ significantly in terms of sex, age, marital status, education, type of surgery, prefracture self-care ability, prefracture walking ability, length of hospital stay, number of comorbid diseases, fracture type, American Society of Anesthesiologists rating, and time from admission to surgery. 
Table 1. Sample Characteristics $(\mathrm{N}=162)$

\begin{tabular}{|c|c|c|c|}
\hline Characteristic & $\begin{array}{l}\text { Intervention } \\
\text { Group }(n=80)\end{array}$ & $\begin{array}{l}\text { Control Group } \\
\quad(n=82)\end{array}$ & $\begin{array}{c}P \text { - } \\
\text { Value }\end{array}$ \\
\hline Age, mean \pm SD & $77.4 \pm 8.2$ & $78.9 \pm 7.3$ & .20 \\
\hline \multicolumn{4}{|l|}{ Sex, n (\%) } \\
\hline Female & $55(68.8)$ & $56(68.3)$ & $>.99$ \\
\hline Male & $25(31.3)$ & $26(31.7)$ & \\
\hline \multicolumn{4}{|l|}{ Marital status, $\mathrm{n}(\%)$} \\
\hline Single & $1(1.3)$ & $0(0)$ & .40 \\
\hline Married & $38(47.5)$ & $46(56.1)$ & \\
\hline Widowed & $40(50)$ & $36(43.9)$ & \\
\hline Divorced & $1(1.3)$ & $0(0)$ & \\
\hline \multicolumn{4}{|l|}{ Educational background, $\mathrm{n}(\%)$} \\
\hline Illiterate & $41(51.3)$ & $38(46.3)$ & .66 \\
\hline Primary school & $22(27.5)$ & $30(36.6)$ & \\
\hline High school & $10(12.5)$ & $8(9.8)$ & \\
\hline College or above & $7(8.8)$ & $6(7.3)$ & \\
\hline \multicolumn{4}{|l|}{ Number of comorbidities, $\mathrm{n}(\%)$} \\
\hline$<2$ & $41(51.3)$ & $52(63.4)$ & .15 \\
\hline$\geq 2$ & $39(48.7)$ & $30(36.6)$ & \\
\hline \multicolumn{4}{|c|}{ American Society of Anesthesiologists rating, $n(\%)$} \\
\hline$<3$ & $45(56.3)$ & $40(48.8)$ & .35 \\
\hline$\geq 3$ & $35(43.7)$ & $42(51.2)$ & \\
\hline \multicolumn{4}{|l|}{ Type of fracture, $n(\%)$} \\
\hline Femoral neck & $51(63.8)$ & $42(51.2)$ & .19 \\
\hline Intertrochanteric & 25 (31.3) & $37(45.1)$ & \\
\hline Subtrochanteric & $4(5)$ & $3(3.7)$ & \\
\hline \multicolumn{4}{|l|}{ Time of surgery, hours $n(\%)$} \\
\hline$<24$ & $28(35)$ & $35(42.7)$ & .11 \\
\hline $24-48$ & $15(18.8)$ & $22(26.8)$ & \\
\hline$>48$ & $37(46.3)$ & $25(30.5)$ & \\
\hline \multicolumn{4}{|l|}{ Type of surgery, $\mathrm{n}(\%)$} \\
\hline Internal fixation & $55(68.8)$ & $47(57.3)$ & .15 \\
\hline Arthroplasty & $25(31.3)$ & $35(42.7)$ & \\
\hline $\begin{array}{l}\text { Length of hospital stay, days, } \\
\text { mean } \pm \text { SD }\end{array}$ & $10.1 \pm 3.7$ & $9.7 \pm 5.0$ & 62 \\
\hline $\begin{array}{l}\text { Patients with independent } \\
\text { prefracture walking ability, } \mathrm{n}(\%)\end{array}$ & $68(85)$ & $69(84.1)$ & $>.99$ \\
\hline $\begin{array}{l}\text { Prefracture self-care ability, } \\
\text { mean } \pm \mathrm{SD}^{\dagger}\end{array}$ & $95.9 \pm 6.6$ & $96.2 \pm 6.4$ & .78 \\
\hline \multicolumn{4}{|c|}{ Recovery to prefracture walking ability, $n$ (\%) } \\
\hline At month 12 & $50(84.7)$ & $44(66.1)$ & .02 \\
\hline At month 18 & $47(81)$ & $27(50.9)$ & .001 \\
\hline At month 24 & $42(75)$ & $28(58.3)$ & .09 \\
\hline \multicolumn{4}{|l|}{ Walking independently, $\mathrm{n}(\%)$} \\
\hline At month 12 & $48(81.4)$ & 39 (62.9) & .03 \\
\hline At month 18 & $43(74.1)$ & $24(45.3)$ & .003 \\
\hline At month 24 & $40(71.4)$ & $24(50.0)$ & .03 \\
\hline \multicolumn{4}{|l|}{ Risk for depressive symptoms, $\mathrm{n}(\%)$} \\
\hline At month 12 & $15(25.9)$ & $24(41.4)$ & .11 \\
\hline At month 18 & $13(23.2)$ & $14(31.8)$ & .37 \\
\hline At month 24 & $15(28.3)$ & $15(35.7)$ & .51 \\
\hline \multicolumn{4}{|c|}{ Geriatric Depression Scale score, mean $\pm \mathrm{SD}^{\ddagger}$} \\
\hline At month 12 & $3.1 \pm 3.2$ & $4.6 \pm 4.3$ & .03 \\
\hline At month 18 & $2.7 \pm 2.7$ & $3.9 \pm 3.9$ & .08 \\
\hline At month 24 & $2.7 \pm 3.3$ & $3.9 \pm 3.6$ & .11 \\
\hline
\end{tabular}

${ }^{*}$ Scores determined according to Chinese Barthel Index (CBI), range 0 (total dependence) to 100 (total independence).

'Ability to perform activities of daily living according to CBI.

${ }^{\ddagger}$ Maximum score 15; higher scores indicating more-severe depressive symptoms.

$\mathrm{SD}=$ standard deviation

\section{Outcome Comparisons}

Analysis of data using the GEE approach showed that subjects in the intervention group had better RHF $(\beta=5.43$, $P<.001$, Table 2) than the control group. For the intervention and control groups, RHF was significantly better at the Months 6, 12, 18, and 24 after discharge than at the Month 1 (Table 2).

The intervention significantly enhanced recovery of walking ability in the intervention group $(\beta=0.80$, $P<.001$, Table 2). As shown in Figure 2, more subjects in the intervention group than in the control group recovered their previous walking ability at different time points during the 24 months after discharge. Overall, the odds ratio for the intervention group recovering their previous walking ability was $2.72(95 \%$ confidence interval $(\mathrm{CI})=11.53-4.84$; $P<.001$ ) compared with the control group (not shown in Table 2). The percentages of subjects recovering walking ability at all time points during the 24 months were significantly higher than during the first month after discharge (Table 2, Figure 2).

Fewer falls occurred in the intervention group than in the control group $(\beta=-0.57, P=.03$, Table 2$)$. Overall, the odds ratio for the intervention group to have a subsequent fall was $0.56(95 \% \mathrm{CI}=0.34-0.94, P=.03)$ compared with the control group (not shown in Table 2). The occurrence of falls did not change significantly over the first year after discharge. More subjects fell from 12 to 24 months after discharge than within the first month. This result might be because of the different observation periods; the latter observation period was 12 months long, whereas the first observation period was only 1 month long (Table 2). The intervention program was not effective in improving the peak force of the quadriceps on the fractured limb and mortality.

The intervention significantly affected self-care ability. Subjects in the intervention group were significantly better at performing ADLs than the control group $(\beta=9.22$, $P<.001$, Table 2 ). The trajectory of CBI scores for the intervention group was consistently better than that of the control group (Table 2, Figure 2). The intervention group improved approximately 8 to 10 points more than the control group on the CBI, or one to two ADLs more, during the first 18 months after discharge and improved approximately 3 points, or 0.5 ADLs more, than the control group from Month 18 to 24 after discharge. For both the intervention and control groups, ADL performance was significantly better at Months 3, 6, 12, 18, and 24 after discharge than at Month 1 (Table 2).

Depressive symptoms decreased significantly over the first 12 months after discharge, especially after the sixth month after discharge (Table 2). Subjects in the intervention group had significantly fewer depressive symptoms than those in the control group $(\beta=-1.31, P=.005$, Table 2$)$. The trajectory of depressive symptoms for the intervention group was consistently lower than that of the control group (Table 2, Figure 3). When Month 1 after discharge was used as baseline, GDS scores significantly decreased at Months 6, 12,18 , and 24 after discharge (Table 2).

For HRQoL, subjects in the intervention group had significantly better PCS than those in the control group $(\beta=6.08, P<.001$, Table 2$)$. The trajectory of PCS for the intervention group was consistently better than that of the 
Table 2. Regression Coefficients of Overall Effects: Time and Intervention

Time After Discharge (Months)

Outcome Variable

Clinical outcomes

Walking recovery

0ccurrence of falls

Ratio of hip flexion

Peak force of quadriceps of fractured limb, pounds

Self-care ability

Activity of daily living performance

Depressive symptoms

Health-related quality of live

Physical component summary scale

Mental component summary scale

Service utilization

Hospital readmissions

Emergency department visits

$\begin{array}{lll}6 & 12 & 18\end{array}$

36

$0.82^{\ddagger}$

0.42

2.64

$2.14^{\ddagger}$

$1.06^{\ddagger}$

0.19

$4.64^{\dagger}$

$3.66^{\ddagger}$

$7.33^{\ddagger}$

7.33
-0.55

$11.08^{\ddagger}$

$-1.03^{\dagger}$

$6.42^{\ddagger}$

6.42
-0.73

$9.78^{\ddagger}$

$-1.75^{*}$

$-0.14$

$-0.24$

\begin{tabular}{l}
0.40 \\
0.35 \\
\hline
\end{tabular}

1

Time uses 1-month data as baseline. Treatment uses control group as baseline. $P<* .05,{ }^{\dagger} .01,{ }^{\ddagger} .001$.

control group (Table 2, Figure 3). PCS increased significantly over the 24 months after discharge (Table 2). The intervention did not affect MCS. When Month 1 was used as baseline, MCS decreased significantly from the Month 6 to 24 (Table 2).

No significant differences were found in 2-year mortality between the two groups (intervention $11.3 \%$ vs control $15.9 \%$ ). Similarly, no significant differences were found
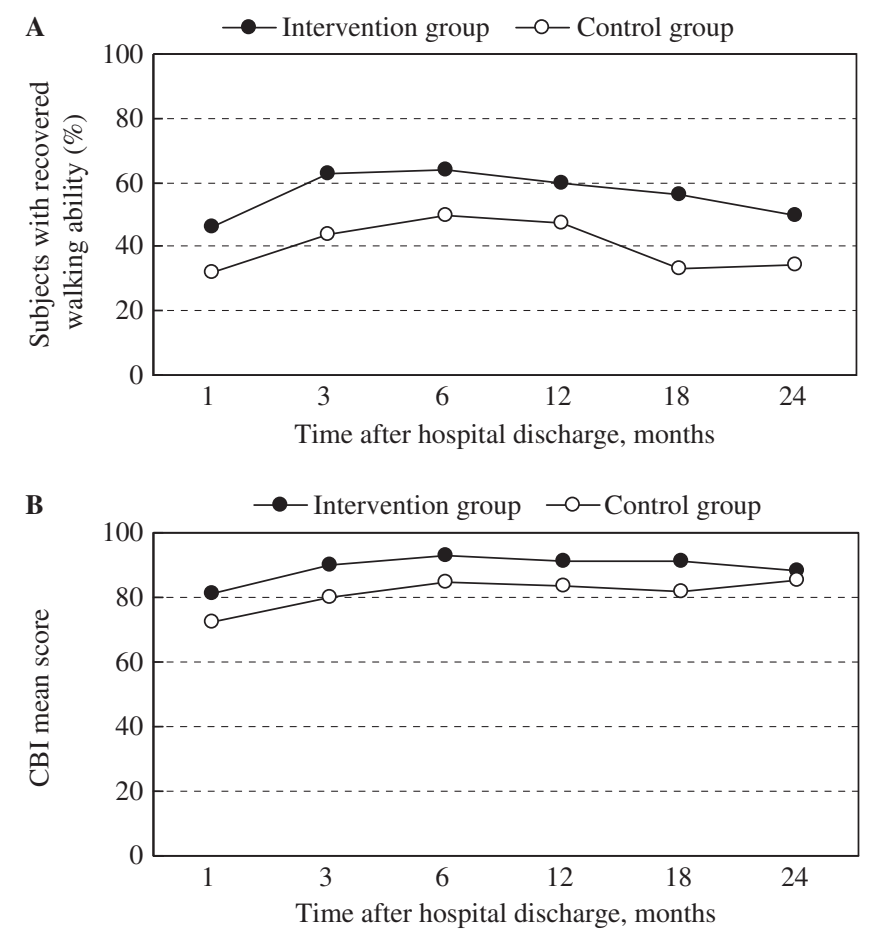

Figure 2. Longitudinal changes in walking ability (A) and selfcare ability (B) for intervention and control groups during second year after discharge. $\mathrm{CBI}=$ Chinese Barthel Index; scores range from 0 (total dependence) to 100 (total independence). in rates of readmission (intervention $11.1 \%$ vs control $7.8 \%$ ) and institutionalization (intervention $2.4 \%$ vs control $2.9 \%$ ) between the two groups. For overall 2-year emergency department visits, the intervention group had
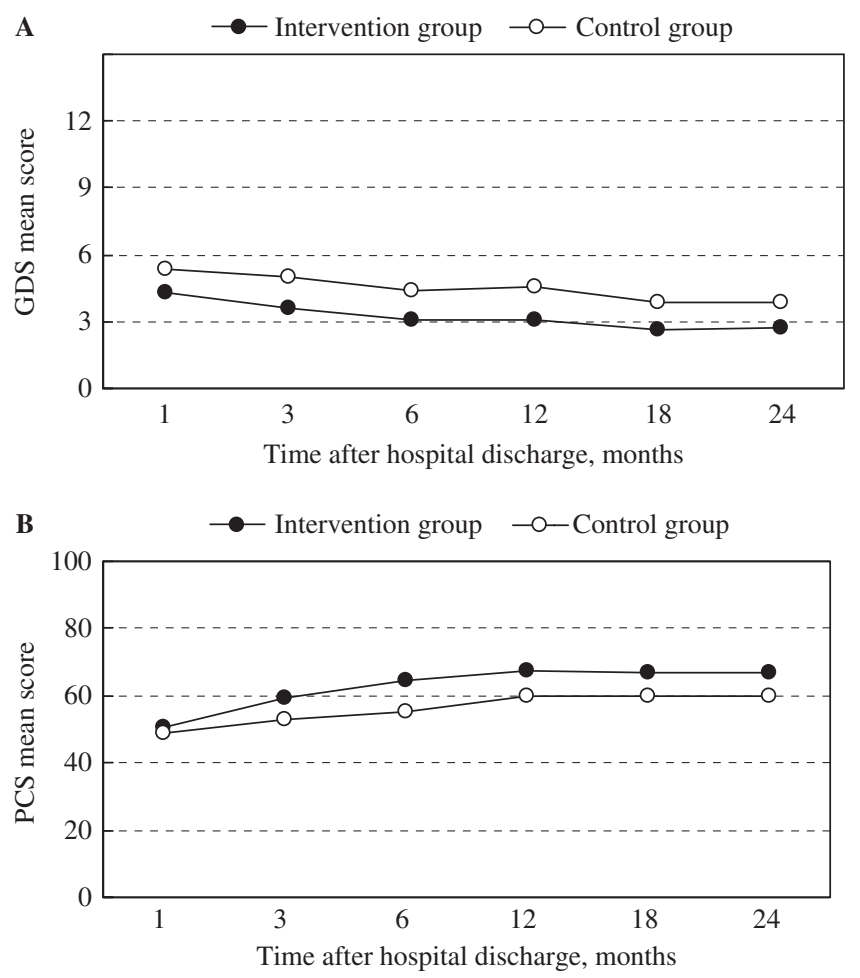

Figure 3. Longitudinal changes in depressive symptoms (A) and health-related quality of life (HRQoL) (B) for intervention and control groups during second year after discharge. GDS $=\mathrm{Ge}-$ riatric Depression Scale; maximum score 15; higher scores indicate more-severe depressive symptoms. PCS = physical component summary scale, range 0-100, higher scores indicate better physical HRQoL. 
significantly fewer visits $(16,4.23 \%)$ than the control group $(32,8.3 \%)($ chi square $=5.34, P=.02)$.

In terms of intervention benefits from the first to second year, similar treatment effects were found at all time points during the 2-year period, including between the first and second year after discharge. This similarity was inferred from the nonsignificant interactions between time and treatment for all outcome variables in the GEE analysis. In other words, as during the first year, the intervention group had significantly better RHF, better performance of ADLs, better recovery of walking and physical health outcomes, fewer falls, and fewer depressive symptoms in the first and the second year after discharge than the control group. Differences between the intervention and control groups were also examined for all variables at single time points, including Months 12, 18, and 24 after discharge, and results were similar to those for the longitudinal analysis. For example, the intervention group had better independence in walking ability and recovery in walking ability than the control group at almost all time points in the first and second years after discharge (Table 1). Although the two groups did not differ significantly in risk for depression at Months 12, 18, and 24 after discharge, the severity of depressive symptoms at these time points was greater in the control group than in the intervention group (Table 1).

\section{DISCUSSION}

This study expands the results of previous studies ${ }^{8,9,11}$ by showing that benefits in self-reported and performancebased outcome indicators of a multidisciplinary program can be maintained in older Taiwanese adults with hip fracture for up to 2 years after discharge. Elderly persons who received the interdisciplinary intervention program had significantly better RHF, better walking ability, fewer subsequent falls, better self-care ability, fewer depressive symptoms, fewer emergency department visits, and better physical health-related outcomes than those who received routine care during the first 2 years after discharge. These results further provide quantitative estimates for the trajectories of treatment effects. Specifically, the treatment effects in the second year after discharge were similar to the treatment effects during the first year.

The multidisciplinary approaches of geriatric hip-fracture programs (GHFPs) and early-support discharge (ESD) programs have been found to effectively increase the rate of return home and reduce length of hospital stay, ${ }^{23}$ but the effect of these two approaches on recovery of mobility and ADLs were not supported. ${ }^{23-25}$ GHFPs are usually implemented within an existing acute orthopedic unit, in which a geriatrician participates and influences care and that has multidisciplinary expertise to provide acute care and rehabilitation. ESD programs are characterized by early identification and transfer of selected patients to their homes from orthopedic units and by expertise in discharge planning, community care, and rehabilitation. Components of GHFPs and ESD programs ${ }^{23}$ were included in the intervention developed and examined in this study and might maximize treatment effects. ${ }^{26}$ Because self-care ability has been found to influence depressive symptoms, ${ }^{27}$ improving self-care ability might indirectly improve depressive symptoms.
This intervention did not significantly affect hospital readmission or mortality rates, as previously reported. ${ }^{23,28}$ Outcomes of death or requirement for institutional care were not found to be affected in a systematic review of nine trials using multidisciplinary approaches to care of older adults with femoral fracture. ${ }^{28}$ The nonsignificant findings might also be due to the small number of rehospitalized and institutionalized patients, as well as the small number of patients who died within the 2-year follow-up.

In terms of changes in outcomes over time, PCS scores and RHF continued to improve during the first 2 years, and MCS continued to decline after Month 6 . These results are consistent with a previous report that different functions were recovered at different rates after hip fracture. ${ }^{7}$ Attention needs to be paid to the decline in overall mental health after the sixth month after discharge and the ineffectiveness of the intervention in improving mental health.

The criteria for selecting subjects excluded older adults with severe cognitive impairment and weak muscle power. Thus, the sample may have had better functional ability than the general population of older adults with hip fracture in Taiwan. The effect of this intervention program can therefore only be generalized for hip-fractured older adults without severe cognitive impairment and with adequate muscle power in their extremities.

Nevertheless, the care received by the control group might be below that provided in most Western countries, postoperatively and especially after discharge. For example, routine care for hip-fractured elderly persons in developed Western countries includes some forms of consultation from different specialists, such as physical or occupational therapy, social work, and geriatric consultation, and most care includes discharge planning and discharge referral for rehabilitation and geriatric services. ${ }^{29}$ These differences may explain why previous consultation interventions have not reported such significant and lasting effects. In addition, some countries have different healthcare delivery systems from Taiwan's. For example, patients with hip fracture in Japan have much longer hospital stays (mean 56.4 days). ${ }^{30}$ Therefore, the effects of the intervention in this study might not be observed if implemented in Western countries or in Japan, limiting the generalizability of the findings to these nations.

Another concern may be that the CBI consisted of observed and self-reported items. Thus, the 0.5 -ADL difference in CBI scores from 18 to 24 months may have marginal clinical significance, particularly in light of the way the instrument was administered. Although the prefracture CBI scores (measured according to subject recall) were obtained differently from CBI scores at other time points (measured according to self-report and observation), the prefracture CBI scores were used for baseline comparison only. The multivariate GEE analysis used only CBI scores collected after discharge, which were measured consistently. In addition, GEE analysis emphasizes mean differences within subjects at different time points. Therefore, the influence of different means of data collection (observation and selfreport) was minimal. Although the 0.5 -ADL difference in CBI scores from 18 to 24 months might have marginal clinical significance, the overall findings based on multiple outcome measures suggest that the treatment effects can be sustained up to 2 years after discharge. 
Another limitation of the study was its single-blind design, in which the personnel delivering the intervention and assessing the outcomes were not blinded, but these personnel were intentionally assigned different research duties to minimize any potential bias. Although some subjects were lost to follow-up in the study period, intentionto-treat and on-protocol analyses had similar results. In conclusion, despite this study's limitations, the interdisciplinary intervention with geriatric hip-fracture program and discharge-support components appeared to benefit elderly persons with hip fracture in Taiwan.

\section{ACKNOWEDGMENTS}

Conflict of Interest: The editor in chief has reviewed the conflict of interest checklist provided by the authors and has determined that the authors have no financial or any other kind of personal conflicts with this paper.

This work was supported by grant NHRI-EX92-9023PL from the National Health Research Institute, Taiwan.

Author Contributions: Yea-Ing L. Shyu: study concept and design, acquisition of subjects and data, analysis and interpretation of data, and preparation of manuscript. Jersey Liang and Min-Chi Chen: study concept and design, analysis and interpretation of data, and preparation of manuscript. Chi-Chuan Wu, Juin-Yih Su, Huey-Shinn Cheng, Shih-Wei Chou, and Ching-Tzu Yang: study concept and design, acquisition of subjects and data, and preparation of manuscript. Ming-Yueh Tseng: analysis and interpretation of data and preparation of manuscript.

Sponsor's Role: The funding agency had no role in the study design, methods, subject recruitment, data collections, analysis, or preparation of manuscript.

\section{REFERENCES}

1. Boonen S, Autier P, Barette $M$ et al. Functional outcome and quality of life following hip fracture in elderly women: A prospective controlled study. Osteoporos Int 2004;15:87-94.

2. Kirke PN, Sutton M, Burke $\mathrm{H}$ et al. Outcome of hip fracture in older Irish women: A 2-year follow-up of subjects in a case-control study. Injury 2002;33:387-391.

3. Magaziner J, Simonsick EM, Kashner TM et al. Predictors of functional recovery one year following hospital discharge for hip fracture: A prospective study. J Gerontol 1990;45:101-107.

4. Rosell PA, Parker MJ. Functional outcome after hip fracture. A 1-year prospective outcome study of 275 patients. Injury 2003;34:529-532.

5. Shyu YIL, Chen MC, Liang J et al. Changes of quality of life among elderly patients with hip fracture in Taiwan. Osteoporos Int 2004;15:95-102.

6. Shyu YI, Chen MC, Liang J et al. Predictors of functional recovery for hip fractured elders at twelve months following hospital discharge: A prospective study on a Taiwanese sample. Osteoporos Int 2004;15:475-482.

7. Magaziner J, Hawkes W, Hebel JR et al. Recovery from hip fracture in eight areas of function. J Gerontol A Biol Sci Med Sci 2000;55A:M498-M507.

8. Adunsky A, Lusky A, Arad M et al. A comparative study of rehabilitation outcomes of elderly hip fracture patients: The advantage of a compre- hensive orthogeriatric approach. J Gerontol A Biol Sci Med Sci 2003;58A: M542-M547.

9. Crotty M, Whitehead CH, Gray S et al. Early discharge and home rehabilitation after hip fracture achieves functional improvements: A randomized controlled trial. Clin Rehabil 2002;16:406-413.

10. Shyu YIL, Liang J, Wu CC et al. A pilot investigation of the short-term effects of an interdisciplinary intervention program on elderly patients with hip fracture in Taiwan. J Am Geriatr Soc 2005;53:811-818.

11. Shyu YI, Liang J, Wu CC et al. Interdisciplinary intervention for hip fracture in older Taiwanese: Benefits last for 1 year. J Gerontol A Biol Sci Med Sci 2008;63A:92-97.

12. Yip PK, Shyu YI, Liu SI et al. The multidisplinary project of dementia study in northern Taiwan (DSNT): Background and methodology. Acta Neurol Sinica 1997;6:210-216.

13. Kraemer HC, Kupfer DJ. Size of treatment effects and their importance to clinical research and practice. Biol Psychiatry 2005;59:990-996.

14. Chen YJ, Dai YT, Yang CT et al. A Review and Proposal on Patient Classification in Long-Term Care System. Taipei, Taiwan: Department of Health, Republic of China, 1995.

15. Lu JR, Tseng HM, Tsai YJ. Assessment of health-related quality of life in Taiwan (I): Development and psychometric testing of SF-36 Taiwan Version. Taiwan J Public Health 2003;22:501-511.

16. Tseng HM, Lu JR, Tsai YJ. Assessment of health-related quality of life (II): Norming and validation of SF-36 Taiwan version. Taiwan J Public Health 2003;22:512-518.

17. Shyu YIL, Lu JF, Liang J. Evaluation of medical outcomes study short form-36 Taiwan version in assessing elderly patients with hip fracture. Osteoporos Int 2004; $15: 575-582$

18. Ware JE, Kosinski MK, Keller SD. SF-36 Physical and Mental Health Summary Scales: A User's Manual. The Health Institute, New England Medical Center, Boston: 1994.

19. Burke WJ, Roccaforte WH, Wengel SP. The short form of the Geriatric Depression Scale: A comparison with the 30-item form. J Geriatr Psychiatry Neurol 1991;4:173-178.

20. Liu CY, Lu CH, Yu S et al. Correlations between scores on Chinese versions of long and short forms of the Geriatric Depression Scale among elderly Chinese. Psychol Rep 1998;82:211-214.

21. Liang KY, Zeger SL. Longitudinal data analysis using generalized linear models. Biometrika 1986;73:13-22.

22. Liang KY, Zeger SL. Regression analysis for correlated data. Annu Rev Public Health 1993;14:43-68.

23. Cameron ID. Coordinated multidisciplinary rehabilitation after hip fracture. Disabil Rehabil 2005;27:1081-1090.

24. Vidan M, Serra JA, Moreno C et al. Efficacy of a comprehensive geriatric intervention in older patients hospitalized for hip fracture: A randomized, controlled trial. J Am Geriatr Soc 2005;53:1476-1482.

25. Ryan T, Enderby P, Rigby AS. A randomized controlled trial to evaluate intensity of community-based rehabilitation provision following stroke or hip fracture in old age. Clin Rehabil 2006;20:123-131.

26. Cameron ID, Crotty M, Currie C et al. Geriatric rehabilitation following fractures in older people: A systematic review. Health Technol Assess $2000 ; 4: 1-121$

27. Lieberman D, Galinsky D, Fried V et al. Geriatric Depression Screening Scale (GDS) in patients hospitalized for physical rehabilitation. Int J Geriatr Psychiatry $1999 ; 14: 549-555$.

28. Cameron ID, Handoll HH, Finnegan TP et al. Co-ordinated multidisciplinary approaches for inpatient rehabilitation of older patients with proximal femoral fractures. Cochrane Database Syst Rev 2001;(3):CD000106.

29. Halbert J, Crotty M, Whitehead C et al. Multi-disciplinary rehabilitation after hip fracture is associated with improved outcome: A systematic review. J Rehabil Med 2007;39:507-512.

30. Committee for Osteoporosis Treatment of the Japanese Orthopaedic Association. Nationwide survey of hip fractures in Japan. J Orthop Sci 2004; $9: 1-5$ 


\section{Appendix A1. Activities for Each Component of the Interdisciplinary Intervention}

\section{Intervention}

Component

Geriatric assessment and consultation

and consultion

Continuous

rehabilitation

\section{Activity (Delivery Person)}

Complete initial assessment of medical and fall history, vita signs, physical examination, physical and cognitive function, nutritional status, preoperative risks, medications, and comorbidities (geriatric nurse)

Geriatric consultation (based on initial assessment) to suggest time of surgery, use of infection and thromboembolic prophylaxis, postoperative nutrition management, urinary tract management, and delirium prevention and management (geriatrician)

Postoperative assessment of signs of delirium, pain, and postoperative complications (geriatric nurse)

Inpatient rehabilitation program: exercise protocol progresses from ankle exercises in bed to knee and hip joint flexion and extension exercises to walking and then climbing up and down stairs using a walker, depending on patient's condition (geriatric nurse, physical therapist, and rehabilitation physician)

At-home rehabilitation program: exercise protocol emphasizes ankle dorsiflexion with knee extension, isometric full knee extension, gently bouncing vertical jump with knee semiflexed and foot on the floor, and ball rolling activities to enhance proprioception, depending on each patient's condition (geriatric nurse and physical therapist)

Discharge planning
Predischarge assessment including caregiver's competence, resources, family function, patient's self-care ability, needs for community or long-term care services, with necessary referrals made (geriatric nurse) Home environmental assessment and suggestions for environmental modifications (geriatric nurse) Telephone calls to remind patient about follow-up visits to clinics (geriatric nurse)

\section{Frequency and Duration of Intervention Activities}

One in-hospital visit approximately 60 minutes before surgery

One in-hospital visit approximately 60 minutes before surgery

One in-hospital visit approximately 30 minutes after surgery

Starts on first day after surgery. One physical therapy session per day with a geriatric nurse ( $\sim 4$ times total, 20 minutes each); two assessments (20 minutes) by a physical therapist; one visit (20 minutes) with a rehabilitation physician

Home nursing visits by geriatric nurse: four visits in first month (once/week, 30 minutes each) and four visits in the second and third months (once every 2 weeks, 30 minutes each). Home visits by physical therapist: one assessment within first week after discharge and at third week and third month after discharge (each 30 minutes). If participants could not work independently during the first 3 months, additional home visits were provided.

One in-hospital visit approximately 30 minutes before discharge, sometimes combined with the in-hospital rehabilitation

One in-home visit approximately 30 minutes before discharge

5-10 minute phone call before each clinic visit at Months 1,3 , and 6 after discharge 